Department of Physics

Papers from the Department of Physics

University of Surrey

Year 2008

\title{
Reduction of spectroscopic strength: Weakly-bound and strongly-bound single-particle states studied using one-nucleon knockout reactions
}
A. Gade
P. Adrich
D. Bazin
M. D. Bowen
B. A. Brown
C. M. Campbell
J. M. Cook
T. Glasmacher
P. G. Hansen
K. Hosier
S. McDaniel
D. McGlinchery
A. Obertelli
K. Siwek
L. A. Riley
J. A. Tostevin, University of Surrey
D. Weisshaar

This paper is posted at Surrey Scholarship Online.

http://epubs.surrey.ac.uk/physicspapers/95 


\title{
Reduction of spectroscopic strength: Weakly-bound and strongly-bound single-particle states studied using one-nucleon knockout reactions
}

\author{
A. Gade ${ }^{1,2}$ P. Adrich, ${ }^{1}$ D. Bazin, ${ }^{1}$ M. D. Bowen,,${ }^{1,2}$ B. A. Brown,,${ }^{1,2}$ C. M. Campbell, ${ }^{1}$ J. M. Cook, ${ }^{1,2}$ T. Glasmacher, ${ }^{1,2}$ \\ P. G. Hansen, ${ }^{1,2}$ K. Hosier, ${ }^{3}$ S. McDaniel,,${ }^{1,2}$ D. McGlinchery, ${ }^{3}$ A. Obertelli, ${ }^{1}$ K. Siwek, ${ }^{1,2}$ L. A. Riley, ${ }^{3}$ J. A. Tostevin, ${ }^{4}$ and \\ D. Weisshaar ${ }^{1}$ \\ ${ }^{1}$ National Superconducting Cyclotron Laboratory, Michigan State University, East Lansing, Michigan 48824, USA \\ ${ }^{2}$ Department of Physics and Astronomy, Michigan State University, East Lansing, Michigan 48824, USA \\ ${ }^{3}$ Department of Physics and Astronomy, Ursinus College, Collegeville, Pennsylvania 19426, USA \\ ${ }^{4}$ Department of Physics, Faculty of Engineering and Physical Sciences, University of Surrey, Guildford, Surrey GU2 7XH, United Kingdom
}

(Received 27 September 2007; published 10 April 2008)

\begin{abstract}
Both one-proton and one-neutron knockout reactions were performed with fast beams of two asymmetric, neutron-deficient rare isotopes produced by projectile fragmentation. The reactions are used to probe the nucleon spectroscopic strengths at both the weakly and strongly bound nucleon Fermi surfaces. The one-proton knockout reactions ${ }^{9} \mathrm{Be}\left({ }^{28} \mathrm{~S},{ }^{27} \mathrm{P}\right) \mathrm{X}$ and ${ }^{9} \mathrm{Be}\left({ }^{24} \mathrm{Si},{ }^{23} \mathrm{Al}\right) \mathrm{X}$ probe the weakly bound valence proton states and the one-neutron knockout reactions and ${ }^{9} \mathrm{Be}\left({ }^{28} \mathrm{~S},{ }^{27} \mathrm{~S}\right) \mathrm{X}$ and ${ }^{9} \mathrm{Be}\left({ }^{24} \mathrm{Si},{ }^{23} \mathrm{Si}\right) \mathrm{X}$ the strongly bound neutron states in the two systems. The spectroscopic strengths are extracted from the measured cross sections by comparisons with an eikonal reaction theory. The reduction of the experimentally deduced spectroscopic strengths, relative to the predictions of shell-model calculations, is of order $0.8-0.9$ in the removal of weakly bound protons and $0.3-0.4$ in the knockout of the strongly bound neutrons. These results support previous studies at the extremes of nuclear binding and provide further evidence that in asymmetric nuclear systems the nucleons of the deficient species, at the more-bound Fermi surface are more strongly correlated than those of the more weakly bound excess species.

DOI: 10.1103/PhysRevC.77.044306

PACS number(s): 24.50.+g, 21.10.Jx, 25.60.Gc
\end{abstract}

\section{INTRODUCTION}

In the nuclear shell model, deeply bound states are usually pictured as being fully occupied by nucleons. For those nucleon states in the vicinity of the Fermi surface, configuration mixing leads to a gradual decrease in the associated occupation numbers. Correlations arising from short-range, soft-core, and tensor nucleon-nucleon $(N N)$ interactions and from longer-range couplings that involve low-lying as well as giant resonance collective excitations result in a further reduction of the physical nucleon occupancies of states near the Fermi surface, the associated single-particle strength being shifted into a large number of states at higher energies [1-3]. Such correlation effects are taken into account only approximately in truncated-model-space effective-interaction theories such as the shell model.

We note that the occupancy of a given state is not an experimental observable. However, these correlation effects are reflected in the distribution of the single-particle spectroscopic strengths. This can be quantified by calculations of the spectroscopic factors $C^{2} S$ and measurements of direct reaction cross sections to given states. Novel measurements of nucleon single-particle strengths can thus be used to quantify the role of correlations. The most quantitative body of data on nucleon strength functions has come from studies of electron-induced proton knockout reactions, $\left(e, e^{\prime} p\right)[1,4]$. It was shown that, in stable nuclei across the nuclear chart, the spectroscopic strengths of valence proton states are reduced by factors $R_{s} \approx 0.6-0.7$ relative to the expectations of the extreme independent-particle model. The correlations are thus revealed as a suppression of the experimental cross sections compared to those based on a dynamical reaction model and an associated nuclear structure model spectroscopic factor. The latter determines the parentage in the initial state of the projectile (with $A$ nucleons) of a specific final-state configuration of a mass $A-1$ residue coupled to a nucleon with quantum numbers $(\ell, j)$ [5]. Information from the $\left(e, e^{\prime} p\right)$ reaction is currently restricted to proton spectroscopic factors and to stable nuclei.

One-nucleon knockout reactions from intermediate-energy projectile beams have already shown an outstanding ability to provide precise spectroscopic information on the dominant proton and neutron single-particle structures of short-lived isotopes far from the valley of $\beta$ stability [6,7]. First employed to study halo systems [8-10], subsequent analyses have used this experimental approach to deduce spectroscopic factors of individual single-particle states in many cases [5,11-16]. In so doing, nucleon knockout reactions have contributed to the clarification of the evolution of shell structure toward the nucleon drip lines, helping to unravel the disappearance of familiar magic numbers and the formation of new shell gaps in nuclei with extreme $N: Z$ ratios [17-22].

At beam energies of $50 \mathrm{MeV} /$ nucleon and higher a theoretical description of the reaction dynamics using the eikonal and the sudden approximations is both appropriate and accurate. There is also a much-reduced model (parameter) dependence compared to analyses of low-energy transfer reactions, e.g., $(p, d)$ and $\left(d,{ }^{3} \mathrm{He}\right)$, whose computation relies on the distorted-waves Born approximation (DWBA) or higher-order formalisms that depend quite sensitively on the optical model and bound-state potentials used in the entrance and exit channels [23]. Such transfer reaction sensitivity is illustrated, for example, by the analyses of Lee et al. 
$[24,25]$. There, depending on the analysis methodology, the benchmark $R_{s} \approx 0.6-0.7$ reduction from the $\left(e, e^{\prime} p\right)$ reactions is reproduced (when using a theoretically consistent set of potential geometries) [24] or is not reproduced (when using a global/generic parameter set) [24,25].

We note that those observables, measured using hadronic reactions, that probe aspects of the spatial behavior and sizes of the nucleonic wave functions are generally in good agreement with the shell-model picture. Examples are the particle angular distributions measured in transfer reactions and the longitudinal momentum distributions of the projectile-like residues measured in heavy-ion induced knockout reactions, both of which identify the orbital angular momentum $\ell$ of the single-particle states involved.

Although the first spectroscopic results from heavy-ioninduced single-nucleon knockout from well-bound nuclei were in line with those found elsewhere $[5,14]$, the reduction factors for the weakly bound protons in ${ }^{8} \mathrm{~B}$ and ${ }^{9} \mathrm{C}$ [26] and the weakly bound neutron in ${ }^{15} \mathrm{C}$ [16] were close to unity, the first indication of a dependence of the reduction on the nucleon separation energy. To probe the analogous spectroscopy of a very strongly bound state, the ${ }^{9} \mathrm{Be}\left({ }^{32} \mathrm{Ar}\right.$, $\left.{ }^{31} \mathrm{Ar}\right) \mathrm{X}$ one-neutron knockout reaction, leading to the only bound state - the $5 / 2^{+}$ground state - of a nucleus at the proton drip line, was performed [15]. The reaction was found to proceed at a cross section of $10.4(13) \mathrm{mb}$, translating into a spectroscopic strength that accounts for only $24(3) \%$ of that predicted by the shell model. It was suggested that this may indeed reflect enhanced correlation effects, absent from the underlying effective-interaction theory, linked to the large asymmetry in the Fermi energies of the neutron and proton states in nuclei near the drip lines [15]. Recent theoretical work, involving a dispersive optical model analysis of nucleon elastic scattering data [27], also indicates that nucleons of the deficient species will become more strongly correlated with increasing asymmetry, whereas the opposite holds for the nucleons of the excess species, in qualitative agreement with the observations reported above [15].

Nuclear systems with a significant nucleon asymmetry and consequent Fermi surface asymmetry can be found along the proton drip line. There, the valence protons are weakly bound and the valence neutrons are strongly bound. In ${ }^{28} \mathrm{~S}$, for example, the relevant separation energies are $S_{p}=2.50 \mathrm{MeV}$ and $S_{n}=21.54 \mathrm{MeV}$. Studies of nucleons near these two, displaced Fermi surfaces is an experimental option that is unique to rare-isotope beam facilities. In addition, rareisotopes produced as fast projectile beams allow the use of thick reaction targets and lead to viable experiments at rates of just a few ions per second.

In the present article we extend the systematics on the measured spectroscopic strengths at the extreme values of nuclear binding. The reactions ${ }^{9} \mathrm{Be}\left({ }^{28} \mathrm{~S},{ }^{27} \mathrm{P}\right) \mathrm{X}$ and ${ }^{9} \mathrm{Be}\left({ }^{24} \mathrm{Si},{ }^{23} \mathrm{Al}\right) \mathrm{X}$ are used to probe the weakly bound proton states and the reactions ${ }^{9} \mathrm{Be}\left({ }^{28} \mathrm{~S},{ }^{27} \mathrm{~S}\right) \mathrm{X}$ and ${ }^{9} \mathrm{Be}\left({ }^{24} \mathrm{Si},{ }^{23} \mathrm{Si}\right) \mathrm{X}$ to probe the strongly bound neutron states. These reactions are particularly well suited because (i) weakly and strongly bound states are probed in the same nucleus and (ii) the knockout residues are located close to the proton drip line and have only one or at most two final states bound against proton emission.

\section{EXPERIMENT}

The projectile beams of ${ }^{24} \mathrm{Si}$ and ${ }^{28} \mathrm{~S}$ were each obtained by fragmentation of a $150 \mathrm{MeV} /$ nucleon ${ }^{36} \mathrm{Ar}$ primary beam provided by the Coupled Cyclotron Facility at the National Superconducting Cyclotron Laboratory (NSCL) on the campus of Michigan State University. The ${ }^{9} \mathrm{Be}$ fragmentation target of thickness $893 \mathrm{mg} / \mathrm{cm}^{2}$ was located at the midacceptance target position of the A1900 fragment separator [28]. A $300-\mathrm{mg} / \mathrm{cm}^{2}$-thick achromatic aluminum wedge degrader and momentum slits at the dispersive image of the fragment separator were employed to purify the beams. ${ }^{24} \mathrm{Si}$ was the most exotic constituent of a cocktail beam of $N=10$ isotones of F, $\mathrm{Ne}, \mathrm{Na}, \mathrm{Mg}, \mathrm{Al}$, and $\mathrm{Si} ;{ }^{28} \mathrm{~S}$ was the most exotic constituent of a cocktail beam of $N=12$ isotones of $\mathrm{Na}, \mathrm{Mg}, \mathrm{Al}, \mathrm{Si}, \mathrm{P}$, and $\mathrm{S}$. These secondary beams contained $0.50 \%{ }^{24} \mathrm{Si}$ and $0.74 \%$ ${ }^{28} \mathrm{~S}$, respectively.

A $188(4)-\mathrm{mg} / \mathrm{cm}^{2}$-thick ${ }^{9}$ Be knockout target was placed at the reaction target position of the S800 spectrograph [29] and surrounded by SeGA (Segmented Germanium Array), a highly segmented germanium detector array optimized for in-beam $\gamma$-ray spectroscopy with fast exotic beams [30]. Its high degree of segmentation allows an accurate event-by-event Doppler reconstruction of the $\gamma$ rays emitted by the reaction residues in-flight, where the emission angle entering the Doppler reconstruction is derived from the location of the detector segment that registered the largest energy deposition. Sixteen of the SeGA detectors were arranged around the target in two rings with central angles of $90^{\circ}$ (nine detectors) and $37^{\circ}$ (seven detectors) relative to the beam axis.

Identification of the projectile-like reaction residues emerging from the target was performed with the focal-plane detector system of the large-acceptance S800 spectrograph [29]. The energy loss measured in the S800 ionization chamber, timeof-flight (TOF) taken between timing scintillators and the position and angle information of the reaction products in the focal plane of the spectrograph were utilized to identify the reaction residues unambiguously on an event-by-event basis. The spectrograph was operated in focus optics mode, where the incoming radioactive beam is focused in momentum at the spectrograph's reaction target position. The time-offlight, measured between two plastic scintillators before the knockout target, provided the particle identification of the incoming beam (see Fig. 1 and Ref. [31]). Software gates applied on the incoming projectiles (Fig. 1, left) allowed for a clean separation between the knockout residues (Fig. 1, right) of interest and the fragmentation products of the different constituents of the cocktail beams.

The two position-sensitive cathode readout drift counters (CRDCs) of the S800 focal-plane detection system in conjunction with the optics code COSY [32] were used to reconstruct the longitudinal momentum distribution of the knockout residues event by event. For each reaction, the inclusive cross sections for the one-nucleon knockout to all bound final states, $\sigma_{\text {inc }}$, is determined from the yield of detected knockout residues divided by the number of incoming projectiles relative to the number density of the ${ }^{9} \mathrm{Be}$ knockout target. A detailed discussion of the cross sections, the residue momentum distributions, and the experimentally deduced spectroscopic strengths will be presented in Sec. IV. 


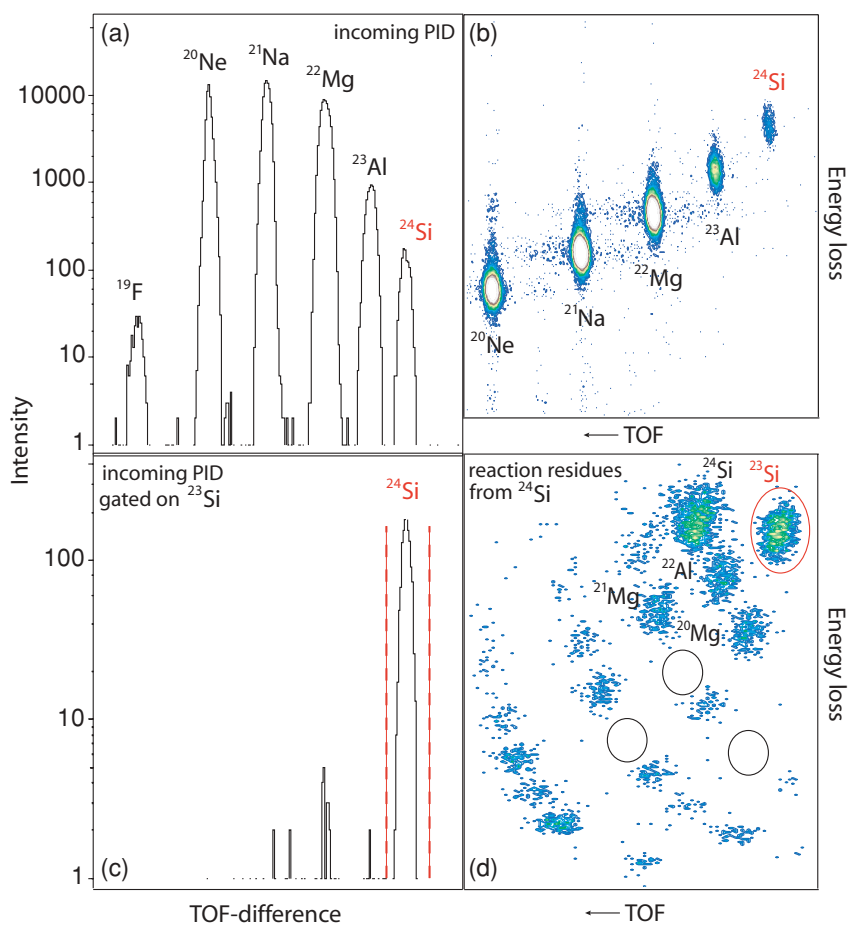

FIG. 1. (Color online) Event-by-event particle identification (PID) spectra of the incoming beam and the reaction residues originating from ${ }^{24} \mathrm{Si}$ projectiles in the exotic cocktail beam. (a) The TOF difference is measured between two timing detectors before the reaction target and the different constituents of the incoming beam are cleanly separated. (b) Energy loss versus TOF. The spectrograph was set to accept the unreacted cocktail beam passing through the target. (d) Reaction residues produced in the collision of ${ }^{24} \mathrm{Si}$ with the ${ }^{9} \mathrm{Be}$ target [software gate applied on the incoming ${ }^{24} \mathrm{Si}$ identified in (a)]. The spectrograph is set to accept the one-neutron knockout residues. Plotted is the ion's TOF versus the energy loss detected in the ion chamber of the S800 spectrograph. The one-neutron knockout residues are well separated from other reaction residues and from the tail of unreacted ${ }^{24} \mathrm{Si}$ projectiles that enter the spectrograph's focal plane. The gaps in this identification matrix correspond to the missing species ${ }^{19} \mathrm{Na}$ and ${ }^{16,15} \mathrm{~F}$, which are already beyond the proton dripline. (c) Same as (a) in coincidence with the ${ }^{23} \mathrm{Si}$ one-neutron knockout residues as identified in (d). It is apparent that with a gate condition as indicated by the dashed lines ${ }^{23} \mathrm{Si}$ reaction residues produced by ${ }^{24} \mathrm{Si}$ are cleanly selected.

\section{THEORETICAL DESCRIPTION}

In one-nucleon knockout reactions, a single nucleon is removed from near the surface of the fast-moving projectile by the light target nucleus, here ${ }^{9} \mathrm{Be}$. The reaction theory is formulated using the sudden and eikonal approximations $[6,33]$. The applicability of the eikonal approximation is linked not only to the high beam energy but also to this surface and small-angle forward scattering dominance of the reaction mechanism [7]. Surface selectivity is ensured by the highly absorptive nature of the residue-target optical potential at the energies under discussion. This surface dominance removes ambiguities associated with (a) the single-nucleon motion and (b) the exit and entrance channel interactions in the nuclear interior, resulting in a much reduced model dependence.
The cross section $\sigma\left(j^{\pi}\right)$ for the removal of a single nucleon with quantum numbers $(\ell, j)$ from the $0^{+}$ground state of an even-even nucleus, thus leaving the knockout residue in a specific final state $j^{\pi}$ with excitation energy $E_{x}\left[j^{\pi}\right]$, is

$$
\sigma\left(j^{\pi}\right)=\left(\frac{A}{A-1}\right)^{2} C^{2} S\left(j^{\pi}\right) \sigma_{\mathrm{sp}}\left(j, S_{N}+E_{x}\left[j^{\pi}\right]\right) .
$$

Here, the sum $S_{N}+E_{x}\left[j^{\pi}\right]$ is the effective separation energy of the removed nucleon and $S_{N}$ is the ground-state-to-groundstate nucleon separation energy. The $A$-dependent term is the required center-of-mass correction to the shell-model spectroscopic factors $C^{2} S$ that is appropriate for the $s d$ shell (the $n=2$ major oscillator shell). The single-particle cross section $\sigma_{\mathrm{sp}}$ is the sum of contributions from both the stripping mechanism (with excitation of the target by the removed nucleon) and the diffractive breakup mechanism (where the target remains in its ground state), written as $\sigma_{\mathrm{sp}}=\sigma_{\mathrm{sp}}^{\mathrm{str}}+\sigma_{\mathrm{sp}}^{\text {dif }}$.

Following Refs. [6,33], these stripping and diffractive contributions are computed independently from the residue- and nucleon-target elastic eikonal $S$ matrices. These are expressed as functions of their respective impact parameters. They are calculated using the double- and single-folding optical limit of Glauber's multiple scattering theory, respectively [34]. The ion-ion, residue-target interactions used the point proton and neutron densities of the residue as input, the densities being taken from Skyrme (SkX) Hartree-Fock (HF) calculations [35]. The ${ }^{9} \mathrm{Be}$ density was assumed to be a Gaussian with a root-mean-squared (rms) radius of $2.36 \mathrm{fm}$. This formalism for treating the nucleon, residue, and target three-body system includes the effects of the breakup of the projectile to all orders, see, e.g., Refs. [6,33].

Given these residue- and nucleon-target interactions, the remaining ingredient to the reaction dynamics is the removednucleon radial overlap or bound-state wave function. As we now show, we must specify consistently the rms radius $r_{\mathrm{sp}}$ of this overlap - that is, the rms radius of the single-nucleon wave function.

Figure 2 shows the single-particle cross sections $\sigma_{\text {sp }}$ calculated for both $1 d_{5 / 2}$ neutron- (circles) and $1 d_{5 / 2}$ proton-removal (squares) from the ${ }^{24} \mathrm{Si}$ beam at $85.3 \mathrm{MeV} /$ nucleon populating the ${ }^{23} \mathrm{Si}$ and ${ }^{23} \mathrm{Al}$ ground states. Each point on the figure uses different radius $r_{0}$ and diffuseness $a_{0}$ parameters in the Woods-Saxon potential well used to calculate the form factor. These parameter values range over $1.1 \leqslant r_{0} \leqslant 1.4 \mathrm{fm}$ and $0.6 \leqslant a_{0} \leqslant 0.8 \mathrm{fm}$. The largest $r_{\mathrm{sp}}$ cases also use a nonlocality parameter $0 \leqslant \beta \leqslant 1.0$. A spin-orbit interaction, with a fixed strength of $6 \mathrm{MeV}$, and with the same $\left(r_{0}, a_{0}\right)$ geometry, is added in each case. Very evident is that the $\sigma_{\mathrm{sp}}$ are determined, to high precision, by the $r_{\mathrm{sp}}$ value of the radial overlap, which must now be assigned.

In our approach to determining the spectroscopic strengths, the removed nucleon-residue relative motion wave functions and their $r_{\mathrm{sp}}$ are also constrained, consistently, by the nuclear and single-particle orbital size systematics of HF calculations using the Skyrme (SkX) interaction parametrization [35]. As was just shown, the knockout cross section calculations are sensitive only to $r_{\mathrm{sp}}$ and are otherwise insensitive to the shape of the binding potential over a large range of parameters. 


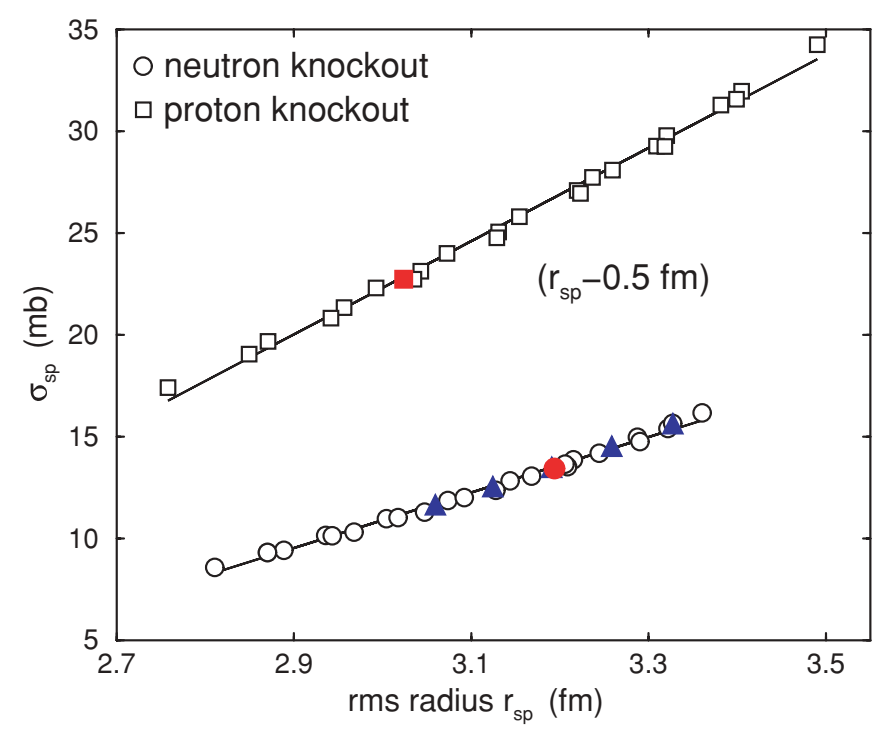

FIG. 2. (Color online) Single-particle cross sections $\sigma_{\mathrm{sp}}$ to the ${ }^{23} \mathrm{Si}$ and ${ }^{23} \mathrm{Al}$ ground states, assuming $1 d_{5 / 2}$ neutron- (circles) and $1 d_{5 / 2}$ proton-removal (squares) from ${ }^{24} \mathrm{Si}$ at $85.3 \mathrm{MeV} /$ nucleon. Each point assumes different radius $r_{0}$ and diffuseness $a_{0}$ parameters for the potential used to calculate the nucleon bound-state wave function. The $r_{\mathrm{sp}}$ values used in the physical calculations are shown by the filled (red) symbols. Cross sections indicated by blue triangles also include a nonlocality $\beta \neq 0$. Note that the proton orbital radii have been displaced to smaller values $\left(r_{\mathrm{sp}}-0.5 \mathrm{fm}\right)$ for display purposes.

For our physical $\sigma_{\text {sp }}$ calculations we thus adopt the following procedure. (1) The bound-state wave functions were calculated in a Woods-Saxon potential with the depth $V_{0}$ adjusted to reproduce the physical separation energy $S_{N}+E_{x}\left(j^{\pi}\right)$ to each final state of interest. (2) The radius parameter $r_{0}$ of this potential well is constrained by the rms radius of this orbital as given by the $\mathrm{HF}$ calculations, $r_{\mathrm{HF}}$. Specifically, we require that $r_{0}$ generates a wave function with $r_{\mathrm{sp}}=[A /(A-1)]^{1 / 2} r_{\mathrm{HF}}$ [15] where this fit is made at the HF-predicted separation energy. (3) Because of the insensitivity to other parameters we use a fixed diffuseness parameter $a_{0}=0.7 \mathrm{fm}$ and a spin-orbit interaction of $6 \mathrm{MeV}$ with the same $\left(r_{0}, a_{0}\right)$ as the central potential. This consistent input, related to nuclear size, is thus mandatory and is included for all of the systems studied. We make use of the SkX Skyrme interaction as it offers good agreement with experiment for several observables directly related to the nuclear size in both stable and exotic nuclei. These include the binding energy differences of mirror nuclei [36], high-energy interaction cross sections [37], and nuclear charge distributions [38]. In Sec. IVC we will compare our calculated results for several different Skyrme parametrizations.

In a limited way, we can also cross reference our procedure for determining $r_{\mathrm{sp}}$ with the values deduced (for even-even stable nuclei in a similar mass range) from $\left(e, e^{\prime} p\right)$ reactions. The $r_{0}$ values used for the analyses of $\left(e, e^{\prime} p\right)$ data are tabulated in Ref. [4]. For example, with the bound-states parameters, nonlocality, and potential conventions adopted there, the resulting $r_{\mathrm{sp}}$ values for ground-state-to-ground-state $\left(e, e^{\prime} p\right)$ knockout from ${ }^{16} \mathrm{O},{ }^{40} \mathrm{Ca}$, and ${ }^{48} \mathrm{Ca}$ are $2.954,3.712$, and

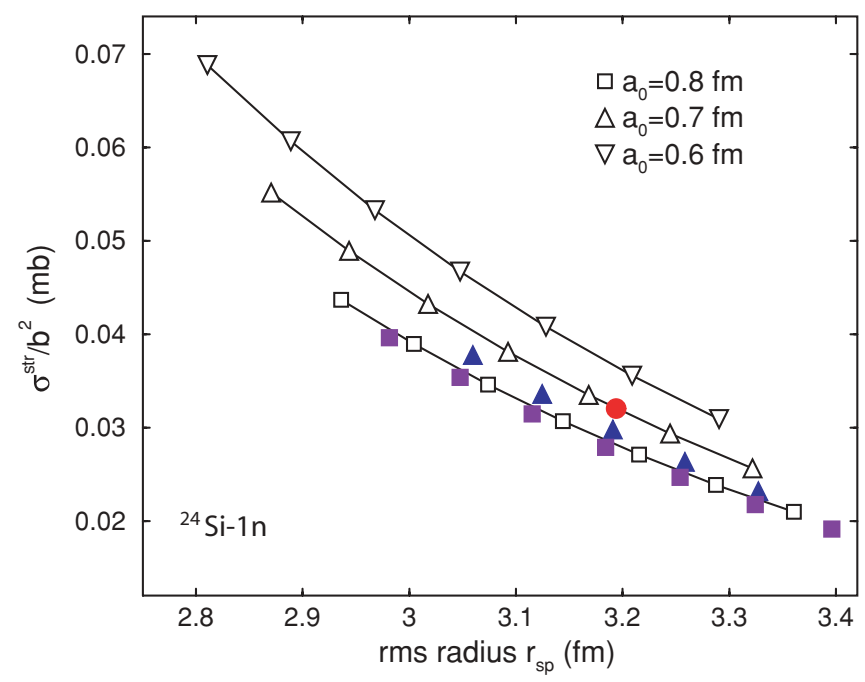

FIG. 3. (Color online) The calculated single particle stripping cross section divided by the square of the asymptotic normalization constant (ANC), $b^{2}$, of the removed nucleon wave function, shown as a function of $r_{\text {sp }}$ for the case of the neutron removal from ${ }^{24} \mathrm{Si}$. The calculations have been performed for a wide range of Woods-Saxon geometries covering a broad range of neutron orbital rms radii. The geometry adopted for our spectroscopic strength discussion is marked by the filled (red) circle. The filled squares and triangles (see the text) correspond to calculations that use nonzero, nonlocality parameters, $\beta$, in the potential. If the reaction calculations were sensitive only to the asymptotic form of the wave functions, then the values of $\sigma^{\text {str }} / b^{2}$ would be equal for all the potential points shown.

$3.58 \mathrm{fm}$, respectively. Based on the HF procedure discussed above, we obtain the values $2.903,3.670$, and $3.56 \mathrm{fm}$, which are in rather good agreement with these cases. For ${ }^{12} \mathrm{C}$, which is rather light for the HF procedure to be expected to work well, the values were 2.77 and $2.63 \mathrm{fm}$ from Ref. [4] and the present approach, respectively.

Figure 3 shows, for the case of the neutron removal from ${ }^{24} \mathrm{Si}$, the single-particle stripping cross section divided by the square of the asymptotic normalization constant (ANC), $b^{2}$. The results, for the wide range of Woods-Saxon potentials that were shown in Fig. 2, are shown as a function of the rms radius of the neutron wave functions, $r_{\mathrm{sp}}$. The filled points are the results for potentials with a nonlocality parameter $\beta>0$, specifically, with $1.1 \leqslant r_{0} \leqslant 1.3 \mathrm{fm}, a_{0}=0.7 \mathrm{fm}, \beta=1.0$ (filled triangles) and with $1.1 \leqslant r_{0} \leqslant 1.4 \mathrm{fm}, a_{0}=0.8 \mathrm{fm}$, $\beta=0.5$ (filled squares). Unlike simply changing the potential geometry, these nonlocal cases exclude some of the wave function from the region of the binding potential and so alter the interior versus exterior content of the neutron wave function. As was discussed in connection with Fig. 2, these nonlocal variations agree with and lie on the (linear) $\sigma_{\mathrm{sp}}$ versus $r_{\text {sp }}$ systematics. As in Fig. 2, the solid circle shows the physical values used here based on our SkX HF prescription. That the knockout reaction cross section is sensitive to more than the tail (the asymptotic form and the ANC) of the neutron wave functions is clear from the dependence of $\sigma^{\text {str }} / b^{2}$ on the potential model assumed. This dependence shows a complex behavior with the radius, diffuseness, and nonlocality parameters. However, as was shown in Fig. 2, this reaction 
sensitivity, critically important such that the cross sections probe more than just the ANC, is encoded effectively and to a high degree of accuracy through the associated value of $r_{\mathrm{sp}}$.

The shape of the longitudinal momentum distribution of the one nucleon knockout residues depends on the $\ell$ value of the removed neutron. The theoretical momentum distributions were calculated following Refs. $[39,40]$ using the same elastic $S$ matrices as were used for the computation of the singleparticle cross sections. These theoretical shapes were folded with the momentum profile of the unreacted projectile beam passing through the target to account for the spread in momenta imposed by (a) the momentum distribution in the incoming beam, (b) its interaction with the target, and (c) the ion optics.

The spectroscopic factors $C^{2} S$ for the reactions studied were calculated in the complete $s d$ shell-model space. New Hamiltonians have recently been obtained for the $s d$ shell [41]. The USDA Hamiltonian was obtained by fitting the 30 most well-determined linear combinations of two-body matrix elements (tbme) and single-particle energies (spe) to 77 ground-state binding energies and 530 excitation energies for nuclei with proton and neutron number between 8 and 20. The starting Hamiltonian as well as the one used for the remaining 46 linear combinations of tbme and spe was the renormalized $G$ matrix obtained from Bonn-A $N N$ potential. The rms deviation between experiment and theory was $170 \mathrm{keV}$. Another Hamiltonian USDB was obtained by fitting 56 linear combinations of tbme and spe with a resulting rms deviation of $137 \mathrm{keV}$. Comparison of results obtained with USDA and USDB provide a measure of the theoretical error associated with the uncertainties in the $s d$-shell part of the Hamiltonian. For the relatively large spectroscopic factors used in this work, the difference between USDA and USDB was less than 5\%; we use the values obtained from USDB for the analysis [41]. All calculations were performed with the computer code OXBASH [42].

\section{RESULTS AND DISCUSSION}

\section{A. Removal of weakly bound protons}

The ${ }^{9} \mathrm{Be}\left({ }^{24} \mathrm{Si},{ }^{23} \mathrm{Al}\right) \mathrm{X}$ and ${ }^{9} \mathrm{Be}\left({ }^{28} \mathrm{~S},{ }^{27} \mathrm{P}\right) \mathrm{X}$ one-proton knockout reactions were performed to measure the single-particle strengths of the weakly bound valence protons.

The ${ }^{9} \mathrm{Be}\left({ }^{24} \mathrm{Si},{ }^{23} \mathrm{Al}\right) \mathrm{X}$ reaction took place at $85.3 \mathrm{MeV} / \mathrm{u}$ midtarget energy. The slits in the A1900 fragment separator were restricted to $0.5 \%$ total momentum acceptance for the projectile beam. The inclusive cross section was measured for several data runs and found to be constant within the statistical uncertainty. The leftmost (low momentum) tail of the longitudinal momentum distribution of the ${ }^{23} \mathrm{Al}$ residues, reconstructed in the focal plane of the S800 spectrograph [see Fig. 4(a)], was cut by the spectrograph's momentum acceptance. A $1.8 \%$ correction for the missing counts was applied to the cross section. The inclusive cross section, including this acceptance correction, amounts to $\sigma_{\text {inc }}=67.3(35) \mathrm{mb}$. A $5 \%$ systematic uncertainty, attributed to fluctuations in the incoming beam composition, was added in quadrature to the statistical uncertainty.

The proton separation energy from ${ }^{23} \mathrm{Al}$ is very low, at $S_{p}=122(19) \mathrm{keV}$. The shell model predicts a first excited
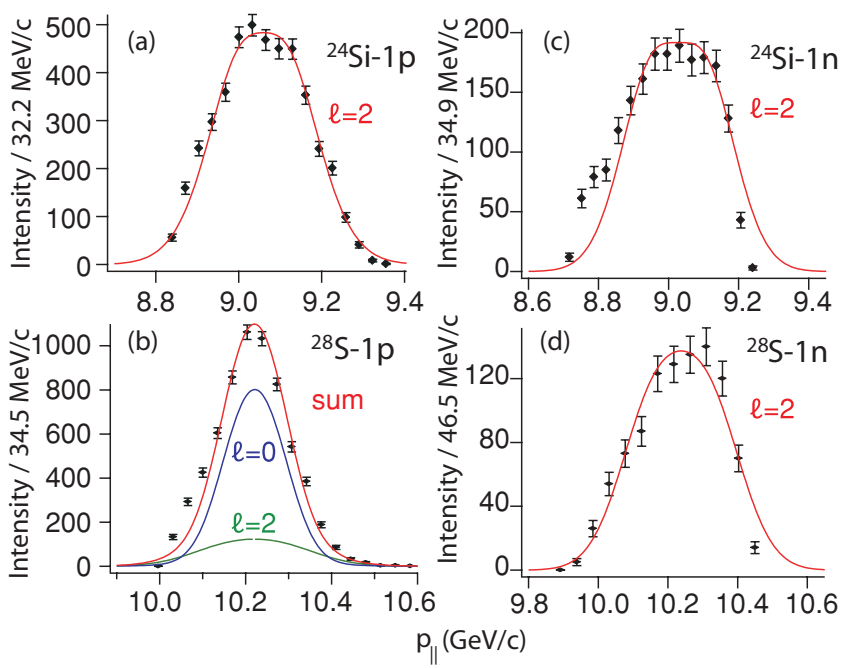

FIG. 4. (Color online) Longitudinal momentum distributions of the knockout residues in the reactions (a) ${ }^{9} \mathrm{Be}\left({ }^{24} \mathrm{Si}^{23} \mathrm{Al}\right) \mathrm{X}$, (b) ${ }^{9} \mathrm{Be}\left({ }^{28} \mathrm{~S},{ }^{27} \mathrm{P}\right) \mathrm{X}$, (c) ${ }^{9} \mathrm{Be}\left({ }^{24} \mathrm{Si},{ }^{23} \mathrm{Si}\right) \mathrm{X}$, and $(\mathrm{d}){ }^{9} \mathrm{Be}\left({ }^{28} \mathrm{~S},{ }^{27} \mathrm{~S}\right) \mathrm{X}$. Calculated shapes are superimposed, pure $\ell=2$ momentum distributions for (a), (c), and (d) and the scaled sum of $\ell=0(80 \%)$ and $\ell=2(20 \%)$ for (b).

state, with $j^{\pi}=1 / 2^{+}$, at $1020 \mathrm{keV}$ and thus the $5 / 2^{+}$ground state is most likely the only state bound against proton emission. The relevant $1 d_{5 / 2}$ proton Woods-Saxon bound-state radius parameter is $r_{0}=1.189 \mathrm{fm}$, given that $r_{\mathrm{HF}}=3.444 \mathrm{fm}$. The ${ }^{24} \mathrm{Si}$ proton separation energy was $S_{p}=3.304 \mathrm{MeV}$. The calculated single-particle cross section is then $\sigma_{\mathrm{sp}}=22.74 \mathrm{mb}$, with 17.56 and $5.18 \mathrm{mb}$ attributed to the stripping and diffraction mechanisms, respectively.

The USDB shell-model calculation predicts the groundstate-to-ground-state spectroscopic factor to be $C^{2} S\left(5 / 2^{+}\right)=$ 3.42. Using Eq. (1), this yields $\sigma^{\text {th }}=84.7 \mathrm{mb}$ to be compared to the experimental value of 67.3(35) $\mathrm{mb}$ and yields a reduction factor $R_{s}=\sigma^{\exp } / \sigma^{\text {th }}=0.79(4)$. Figure $4($ a) compares the measured ${ }^{23} \mathrm{Al}$ residue longitudinal momentum distribution to the shape calculated for the removal of a proton from the $1 d_{5 / 2}$ orbit, using the model of Refs. $[39,40]$. The agreement is good and confirms the orbital angular momentum assignment for the removed proton and the expectation that the measured inclusive cross section is exhausted by knockout to the ground state of ${ }^{23} \mathrm{Al}$.

The ${ }^{9} \mathrm{Be}\left({ }^{28} \mathrm{~S},{ }^{27} \mathrm{P}\right) \mathrm{X}$ reaction was performed at $80.7 \mathrm{MeV} / \mathrm{u}$ at $1 \%$ incoming momentum spread of the projectile beam. The inclusive cross section was measured for several data runs and found to be constant within the statistical uncertainty. However, during three normalization runs, the fraction of ${ }^{28} \mathrm{~S}$ in the incoming cocktail was found to change from 0.735 to $0.818 \%$ and back to $0.751 \%$. A composition change is not unusual in experiments with cocktail beams, especially when the isotope of interest is the least abundant constituent, as in this case. This uncertainty in the normalization requires an $8.7 \%$ systematic uncertainty to be added in quadrature to the statistical uncertainty. A momentum-acceptance correction of $1.78 \%$ has been applied to the inclusive cross section to account for the finite momentum acceptance of the S800 spectrograph and the loss of counts at the outermost tails 


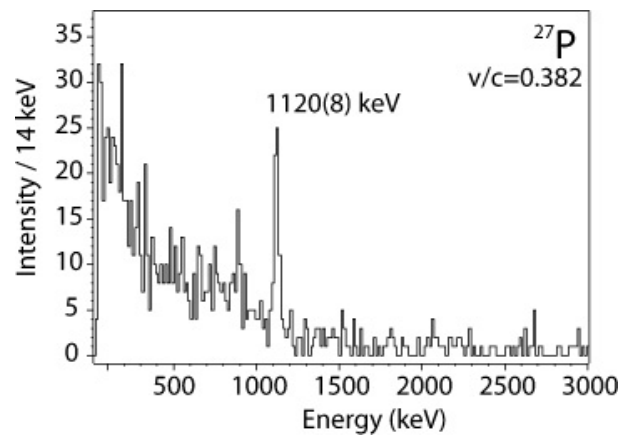

FIG. 5. $\gamma$-ray spectrum detected in coincidence with ${ }^{27} \mathrm{P}$. The $1120-\mathrm{keV}$ level is populated to $18(3) \%$ in the one-proton knockout from ${ }^{28} \mathrm{~S}$. According to the shell model, one would expect $40 \%$. The discrepancy is most likely explained by the fact that this level is unbound by $260 \mathrm{keV}$ and the $\gamma$-ray decay will be in strong competition with proton emission. The $3 / 2^{+}$state was previously reported at $1199 \mathrm{keV}$ from particle spectroscopy [44].

[see Fig. 4(b)]. The inclusive one-proton knockout cross section to ${ }^{27} \mathrm{P}$, including the acceptance correction, statistical, and systematic uncertainties is $\sigma_{\text {inc }}=38(4) \mathrm{mb}$.

The USDB shell-model calculations (in agreement with the mirror nucleus) predict a ${ }^{27} \mathrm{P}$ first excited state with spin and parity $3 / 2^{+}$at $990 \mathrm{keV}$, just above the proton separation energy of 861(27) keV [43]. However, in qualitative agreement with the high $3 / 2^{+}$excitation energy reported in Ref. [44], we observed a $\gamma$-ray transition at $1120(8) \mathrm{keV}$, attributed to the de-excitation of the first excited state to the $1 / 2^{+}$ground state. Figure 5 displays the $\gamma$-ray spectrum as detected in SeGA and event-by-event Doppler reconstructed into the projectile rest frame.

The single-particle cross section for the knockout to the $1 / 2^{+}$ground state of ${ }^{27} \mathrm{P}$ required a bound-state potential with $r_{0}=1.046 \mathrm{fm}$ based on $r_{\mathrm{HF}}=3.895 \mathrm{fm}$. The ground- state proton separation energy was $S_{p}\left({ }^{28} \mathrm{~S}\right)=2.5 \mathrm{MeV}$ [43]. The calculated single-particle cross section is then $\sigma_{\mathrm{sp}}=$ $28.57 \mathrm{mb}$, with 20.73 and $7.84 \mathrm{mb}$ attributed to the stripping and diffraction mechanisms. The one-proton removal cross section to the $3 / 2^{+}$excited state used $r_{0}=1.239 \mathrm{fm}$, based on $r_{\mathrm{HF}}=3.489 \mathrm{fm}$. The calculated $3 / 2^{+}$state single-particle cross section is then $\sigma_{\mathrm{sp}}=19.01 \mathrm{mb}$.

The USDB shell-model calculations predict spectroscopic factors of $C^{2} S\left(1 / 2^{+}\right)=0.832$ and $C^{2} S\left(3 / 2^{+}\right)=0.820$ for these lowest-lying $1 / 2^{+}$and $3 / 2^{+}$states. So when combined with the single- particle cross sections these yield a total theoretical cross section of $\sigma^{\text {th }}=\sigma^{\text {th }}\left(1 / 2^{+}\right)+\sigma^{\text {th }}\left(3 / 2^{+}\right)=$ $42.3 \mathrm{mb}$. When compared with the experimental inclusive cross section we obtain a reduction factor of $R_{s}=0.90(7)$. In the future, threshold effects introduced by the proximity of the particle continuum [45] might be taken into account within the novel Gamow shell model [46] or other approaches that explicitly treat the continuum [47-50].

From the efficiency-corrected peak area of the $\gamma$-ray transition at $1120 \mathrm{keV}$ relative to the number of knockout residues we determined the population of the $3 / 2^{+}$excited final state of ${ }^{27} \mathrm{P}$ relative to the inclusive cross section to be $18(3) \%$. This is to be compared to a theoretically predicted population of $40 \%$ from the combination of shell model and reaction theory. The lower measured branch is most likely attributed to a significant competition from proton decay of this state located at about $260 \mathrm{keV}$ above the proton threshold.

The experimental inclusive momentum distribution is overlaid with calculated $\ell=0$ and $\ell=2$ shapes in Fig. 4(b). These $\ell=0$ and $\ell=2$ shapes have been scaled so that their integrated values are in the ratio $20 \% \ell=2$ and $80 \% \ell=0$, as suggested from the experimental $\gamma$-ray yield. The shape calculated in this way matches the data. These experimental and theoretical results are collected in Table $\mathrm{I}$. The ${ }^{23} \mathrm{Al}(\mathrm{gs})$

TABLE I. Summary of the results for the one-proton and one-neutron knockout from ${ }^{24} \mathrm{Si}$ and ${ }^{28} \mathrm{~S}$ projectiles. Given are the excitation energy of the final states in the projectile-like knockout residues, the spin and parity, the experimental branching ratios, the measured cross sections, the shell-model single-particle orbitals, the single-particle cross sections from the eikonal theory and their composition into stripping and diffractive contributions, the shell-model spectroscopic factors (USDB effective interaction), the resulting theoretical cross sections from Eq. (1), the theoretical branching ratios, and the deduced reduction factors.

\begin{tabular}{|c|c|c|c|c|c|c|c|c|c|c|c|c|}
\hline Res. & $\begin{array}{c}E_{x} \\
(\mathrm{keV})\end{array}$ & $\begin{array}{l}J^{\pi} \\
(\hbar)\end{array}$ & $\begin{array}{c}\mathrm{BR}^{\exp } \\
(\%)\end{array}$ & $\begin{array}{c}\sigma \\
(\mathrm{mb})\end{array}$ & $\begin{array}{c}\text { Conf. } \\
\text { SM }\end{array}$ & $\begin{array}{c}\sigma_{\mathrm{sp}} \\
(\mathrm{mb})\end{array}$ & $\begin{array}{c}\sigma_{\mathrm{sp}}^{\mathrm{str}} \\
(\mathrm{mb})\end{array}$ & $\begin{array}{c}\sigma_{\mathrm{sp}}^{\mathrm{dif}} \\
(\mathrm{mb})\end{array}$ & $\begin{array}{l}C^{2} S \\
\mathrm{SM}\end{array}$ & $\begin{array}{c}\sigma^{\text {th }} \\
(\mathrm{mb})\end{array}$ & $\begin{array}{c}\mathrm{BR}^{\text {th }} \\
(\%)\end{array}$ & $R_{s}$ \\
\hline \multicolumn{13}{|c|}{ Projectile ${ }^{24} \mathrm{Si}$} \\
\hline${ }^{23} \mathrm{Al}$ & 0 & $5 / 2^{+}$ & 100 & $67.3(35)$ & $d_{5 / 2}$ & 22.74 & 17.56 & 5.18 & 3.42 & 84.68 & 100 & $0.79(4)$ \\
\hline${ }^{23} \mathrm{Si}$ & 0 & $5 / 2^{+}$ & 100 & $9.8(10)$ & $d_{5 / 2}$ & 13.43 & 10.96 & 2.47 & 1.71 & 25.01 & 100 & $0.39(4)$ \\
\hline \multicolumn{13}{|c|}{ Projectile ${ }^{28} S$} \\
\hline${ }^{27} \mathrm{P}$ & 0 & $1 / 2^{+}$ & $82(7)$ & $31(3)$ & $s_{1 / 2}$ & 28.57 & 20.73 & 7.84 & 0.832 & 25.56 & 60.4 & \\
\hline & 1100 & $3 / 2^{+}$ & $18(3)$ & $6.8(11)$ & $d_{3 / 2}$ & 19.01 & 14.61 & 4.40 & 0.82 & 16.76 & 39.6 & \\
\hline Inc. & & & & $38(3)$ & & & & & & 42.32 & & $0.90(7)$ \\
\hline \multirow[t]{2}{*}{${ }^{27} \mathrm{~S}$} & 0 & $5 / 2^{+}$ & & & $d_{5 / 2}$ & 11.09 & 8.99 & 2.10 & 3.136 & 37.40 & 96.5 & \\
\hline & $\leqslant 100$ & $3 / 2^{+}$ & & & $d_{3 / 2}$ & 10.75 & 8.72 & 2.03 & 0.119 & 1.37 & 3.5 & \\
\hline Inc. & & & & $11.9(12)$ & & & & & & 38.77 & & $0.31(3)$ \\
\hline
\end{tabular}


single-particle cross-section calculation is shown by the (red) filled square in Fig. 2.

\section{B. Removal of strongly bound neutrons}

The ${ }^{9} \mathrm{Be}\left({ }^{24} \mathrm{Si},{ }^{23} \mathrm{Si}\right) \mathrm{X}$ and ${ }^{9} \mathrm{Be}\left({ }^{28} \mathrm{~S},{ }^{27} \mathrm{~S}\right) \mathrm{X}$ one-neutron knockout reactions were performed to measure the spectroscopic strengths for the removal of the strongly bound neutrons from these proton-excess nuclei.

The ${ }^{9} \mathrm{Be}\left({ }^{24} \mathrm{Si},{ }^{23} \mathrm{Si}\right) \mathrm{X}$ reaction was carried out at $85.3 \mathrm{MeV} / \mathrm{u}$ midtarget energy. The momentum acceptance of the A1900 was restricted to $0.5 \%$. The data were divided up into clusters covering about $4 \mathrm{~h}$ of run time each. The inclusive cross section was determined cluster by cluster and found to be constant within the statistical uncertainty. An inclusive cross section of $\sigma_{\text {inc }}=9.8(10) \mathrm{mb}$ was derived. The error includes a $10 \%$ systematic uncertainty attributed to a possible acceptance cut, seen on the high-energy side of the parallel momentum distribution in Fig. 4(c). Unlike the cases described in the previous section, the distribution is seen to be asymmetric.

No $\gamma$-ray transition, which would indicate population of an excited state in ${ }^{23} \mathrm{Si}$, was observed. The first excited state of ${ }^{23} \mathrm{Si}$, with $j^{\pi}=1 / 2^{+}$, is predicted to be at $1780 \mathrm{keV}$ and so is probably above the proton separation energy of $S_{p}=$ $1700(220) \mathrm{keV}$. Unlike in ${ }^{27} \mathrm{P}$, where the $3 / 2^{+}$state above the proton separation energy has orbital angular momentum $\ell=2$ and its proton decay is thus hindered by the centrifugal barrier, the excited state in ${ }^{23} \mathrm{Si}$ has orbital angular momentum $\ell=0$ and so its proton emission would not be hindered by a centrifugal barrier.

The single-particle cross section for the population of the $5 / 2^{+}$ground state was calculated using $r_{0}=1.317 \mathrm{fm}$, consistent with the HF $1 d_{5 / 2}$ neutron orbital rms radius $r_{\mathrm{HF}}=3.237 \mathrm{fm}$. The neutron separation energy from ${ }^{24} \mathrm{Si}$ was $S_{n}=21.09 \mathrm{MeV}$ [43]. The calculated stripping and diffractive cross-section contributions were $\sigma_{\mathrm{sp}}^{\mathrm{str}}=10.96 \mathrm{mb}$ and $\sigma_{\mathrm{sp}}^{\text {dif }}=2.47 \mathrm{mb}$, giving a theoretical single-particle cross section of $\sigma_{\mathrm{sp}}=13.43 \mathrm{mb}$ to the ground state of ${ }^{23} \mathrm{Si}$.

The USDB shell-model calculations predict a spectroscopic factor of $C^{2} S\left(5 / 2^{+}\right)=1.71$. This gives $\sigma^{\text {th }}=25.01 \mathrm{mb}$, to be compared to the experimental value of $\sigma_{\text {inc }}=9.8(10) \mathrm{mb}$. Similar to the one-neutron removal from ${ }^{32} \mathrm{Ar}$ to the ground state of ${ }^{31} \mathrm{Ar}$ [15], the result implies a strong reduction, with $R_{s}=0.39(4)$. In Fig. 4(c) the experimental longitudinal momentum distribution is compared to the calculation for the removal of an $\ell=2$ neutron from ${ }^{24} \mathrm{Si}$. The experiment and calculations agree well except for the extreme high-momentum tail where the experimental distribution appears to be cut off.

The ${ }^{9} \mathrm{Be}\left({ }^{28} \mathrm{~S},{ }^{27} \mathrm{~S}\right) \mathrm{X}$ reaction was performed at $80.7 \mathrm{MeV} / \mathrm{u}$ at $1 \%$ incoming momentum spread of the projectile beam. The inclusive cross sections, measured for several data runs, were constant within the statistical uncertainty. The measured inclusive cross section is $\sigma_{\text {inc }}=11.9(12) \mathrm{mb}$. Similar to the one-neutron removal case above, a $10 \%$ systematic uncertainty was added in quadrature to account for a possible acceptance cut seen on the high-energy side of the parallel momentum distribution [Fig. 4(d)]. The absolute cross sections agree within $0.9 \%$ for two different normalization runs and hence no additional systematic uncertainty was added for normalization of the incoming beam.

Two bound final states are expected in the ${ }^{27} \mathrm{~S}$ knockout residue below the proton separation energy of $S_{p}=$ $720(280) \mathrm{keV}$, the $5 / 2^{+}$ground state and a $3 / 2^{+}$first excited state. The USDB shell model predicts these states to be almost degenerate. In the $\gamma$-ray spectrum, taken in coincidence with ${ }^{27} \mathrm{~S}$, a weak low-energy transition cannot be excluded but its identification is obscured by background and hampered by low statistics. As outlined below, this is consistent with the level ordering proposed by the shell model.

The single-particle cross section for the knockout to the $5 / 2^{+}$ground state was calculated with $r_{0}=1.308 \mathrm{fm}$ for the neutron $1 d_{5 / 2}$ orbit, based on $r_{\mathrm{HF}}=3.300 \mathrm{fm}$. The groundstate neutron separation energy was $S_{n}=21.54$ [43]. This gives a single-particle cross section to the $d_{5 / 2}$ ground state of $\sigma_{\mathrm{sp}}\left(5 / 2^{+}\right)=11.09 \mathrm{mb}$, with 8.99 and $2.10 \mathrm{mb}$ attributed to stripping and diffraction. The single-particle cross section to the $3 / 2^{+}$state was calculated in the same way, but with $r_{0}=$ $1.354 \mathrm{fm}$, giving $\sigma_{\mathrm{sp}}\left(3 / 2^{+}\right)=10.75 \mathrm{mb}$, and with 8.72 and $2.03 \mathrm{mb}$ attributed to the stripping and diffraction mechanisms.

The USDB shell-model calculations predict $C^{2} S\left(5 / 2^{+}\right)=$ 3.136 and $C^{2} S\left(3 / 2^{+}\right)=0.119$. Combining with the single-particle cross sections, $\sigma^{\text {th }}=\sigma^{\text {th }}\left(5 / 2^{+}\right)+\sigma^{\text {th }}\left(3 / 2^{+}\right)=$ $38.77 \mathrm{mb}$ with only $3.5 \%$ expected to populate the excited state (consistent with its nonobservation in the experiment). A strong reduction factor $R_{s}=\sigma^{\exp } / \sigma^{\text {th }}=0.31(3)$ results. In Fig. 4(d) the experimental longitudinal momentum distribution is again compared to a calculated shape for the removal of $\ell=2$ neutrons, corresponding to knockout to both $d_{5 / 2}$ and $d_{3 / 2}$ final states. Except for the high-momentum tail, the calculation agrees well with the experiment, suggesting that only the $3 / 2^{+}$and $5 / 2^{+}$states in ${ }^{27} \mathrm{~S}$ are populated. These experimental and theoretical results are also collected in Table I. The ${ }^{23} \mathrm{Si}$ (gs) single-particle cross section calculation is also shown by the (red) filled circle in Fig. 2.

We note that the high neutron separation energy involved in these cases does not influence on the underlying knockout reaction mechanism. This is unlike the situation in transfer reactions where the surface localization and discrete initial and final states of the reaction can generate significant sensitivity of the transition amplitude to the extent of linear and angular momentum (mis-)matching, and which leads to semiclassical conditions for well-matched transfer cross sections, e.g., Ref. [51]. The (dominant) stripping and elastic breakup knockout mechanisms are inclusive with respect to the final states of the target and projectile, respectively. The main assumption of the eikonal theory, that the core (residue) nucleus travels with constant velocity throughout the collision, is well satisfied at energies of order $100 \mathrm{MeV}$ per nucleon and is verified empirically by the measured centroids of the residue parallel momentum distributions.

\section{Discussion}

Figure 6 includes the present results on a plot of the systematics of the measured reduction factors $R_{S}$ as a function 


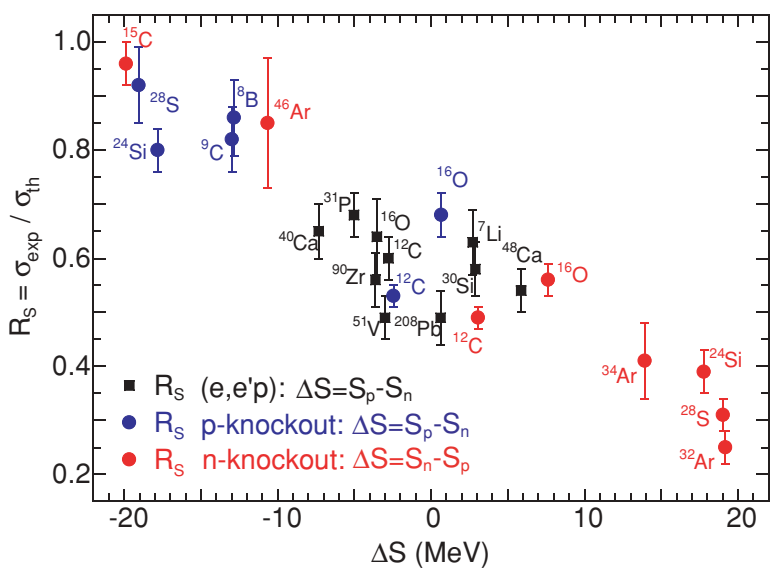

FIG. 6. (Color online) Reduction of the measured nucleon knockout cross sections (spectroscopic strength) relative to theoretical values as a function of the difference in separation energies of the two nucleon species, $\Delta S$ (see text). The data points are from Refs. [5,13-16,19,24]. Those from the present work, labeled ${ }^{24} \mathrm{Si}$ and ${ }^{28} \mathrm{~S}$, appear on the extreme left- and right-hand sides of the figure. Only experimental uncertainties are included.

of the differences in separation energies of the deficient and excess nucleon species in the projectile, $\Delta S$. For proton removal we define $\Delta S=S_{p}-S_{n}$ and for neutron removal $\Delta S=S_{n}-S_{p}$, where $S_{n}$ and $S_{p}$ are the effective nucleon separation energies. The quantity $\Delta S$ is a measure of the asymmetry of the Fermi surfaces in each nucleus. $\Delta S$ takes on large negative values for reactions where a weakly bound nucleon of the excess species is removed and large positive values for reactions where a strongly bound nucleon of the deficient species is removed.

The plot includes data points from both heavy-ion-induced one-proton and one-neutron knockout reactions and from the electron-induced proton removal from stable nuclei. Unlike the earlier comparisons of the $\left(e, e^{\prime} p\right)$ spectroscopic strengths with the extreme independent-particle model, that yield factors $R_{s} \approx 0.6-0.7$, here we compare with shell-model spectroscopic factors, as was carried out in Ref. [24]. Near $\Delta S=0$ - the stable and well-bound systems - the values cluster around reduction factors $R_{s} \approx 0.5-0.7$, with heavy-ion and electron-induced knockout in agreement. At the extremes of nuclear binding, reduction factors $R_{s} \approx 0.25-0.40$ are found in the removal of a nucleon of the deficient species [e.g., the results from the present study of $\left({ }^{24} \mathrm{Si},{ }^{23} \mathrm{Si}\right)$ and $\left({ }^{28} \mathrm{~S},{ }^{27} \mathrm{~S}\right)$, whereas the reduction factors are much closer to unity, with $R_{s} \approx 0.80-1.0$, when the removed nucleon is in excess (e.g., the results from the present study of $\left({ }^{24} \mathrm{Si},{ }^{23} \mathrm{Al}\right)$ and $\left.\left({ }^{28} \mathrm{~S},{ }^{27} \mathrm{P}\right)\right]$. The results of the present work fit nicely into the existing systematics and give additional support to the suggestion that the strength of correlation effects, missing to an (as yet) unknown extent from effective interaction theories - here the shell model - depend on the asymmetry of the two nucleon Fermi surfaces. The present work suggests an enhancement of the correlation effects experienced by strongly bound valence nucleons of the deficient type and weakened correlations of the excess nucleons at the weakly bound Fermi surface.

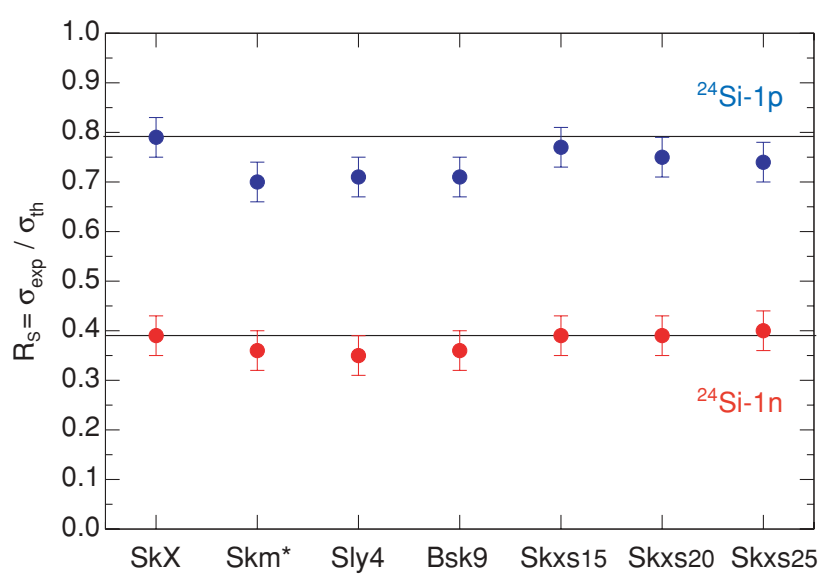

FIG. 7. (Color online) Deduced values of $R_{s}$ for the reactions ${ }^{9} \mathrm{Be}\left({ }^{24} \mathrm{Si},{ }^{23} \mathrm{Al}\right) \mathrm{X}$ and ${ }^{9} \mathrm{Be}\left({ }^{24} \mathrm{Si},{ }^{23} \mathrm{Si}\right) \mathrm{X}$ as obtained using different Skyrme parametrizations as input to the HF calculations used for the reaction methodology. The $R_{s}$ factors obtained when using the Skm*, Sly4, Bsk9, Skxs15, Skxs20, and Skxs25 interactions agree within the quoted uncertainties on the value deduced using the SkX Skyrme parametrization used here. The $\mathrm{SkX}$ values are indicated by the horizontal lines.

Finally, we address the sensitivity of the reaction methodology to details of the Skyrme interaction used to constrain the residue densities and the rms radii $r_{\mathrm{sp}}$ of the wave functions of the removed nucleons. Figure 7 shows the deduced suppression factors $R_{s}$ for the reactions ${ }^{9} \mathrm{Be}\left({ }^{24} \mathrm{Si}\right.$, $\left.{ }^{23} \mathrm{Al}\right) \mathrm{X}$ and ${ }^{9} \mathrm{Be}\left({ }^{24} \mathrm{Si},{ }^{23} \mathrm{Si}\right) \mathrm{X}$ for several different Skyrme parametrizations, including the $\mathrm{SkX}$ model, favored here.

As mentioned in Sec. III, we use the SkX Skyrme interaction [35] for the nuclear densities and single-particle rms radii because it has been extensively tested with regard to size and binding energy observables [36-38]. But there are other Skyrme parameter sets available. The main difference between them can be related to the nuclear-matter incompressibility $K$ and the slope of the neutron equation-of-state near nuclearmatter density $P_{n} . P_{n}$ is correlated with the neutron-skin thickness in nuclei with $N \neq Z$ [52] and hence can be a source of uncertainty for the densities and single-particle radii in nuclei far from stability. The SkX interaction has a relatively large incompressibility, $K=270 \mathrm{MeV}$, and a neutron skin of $T=r_{n}-r_{p}=0.16 \mathrm{fm}$ for ${ }^{208} \mathrm{~Pb}$, where $r_{p / n}$ is the rms radius for protons/neutrons. Thus, we need to test the sensitivity of our results to reasonable variations in the Skyrme parameters related to these quantities. The results for one-proton and one-neutron removal from ${ }^{24} \mathrm{Si}$ are shown in Fig. 7. Skm* [53] is used because it gives a slightly better surface diffuseness for the charge density $[37,54]$ compared to SkX. This change can be traced to a smaller nuclear matter incompressibility, which is smaller for Skm* $(K=215 \mathrm{MeV})$ compared to SkX. The recent Skxs15, Skxs20, and Skxs25 Skyrme interactions [54] represent a reasonable variation of neutron-skin thickness in ${ }^{208} \mathrm{~Pb}$ [52], with $T=0.15,0.20$, and $0.25 \mathrm{fm}$, respectively, and all have $K=200 \mathrm{MeV}$. We also compare to results with the widely used Sly4 interaction [55] ( $K=230 \mathrm{MeV}$ and $T=0.16 \mathrm{fm}$ ) and with the Bsk9 interaction [56] obtained from a recent global fit to binding energies together 
with the Friedman-Pandharipande prediction for $P_{n}(K=$ $230 \mathrm{MeV}$ and $T=0.16 \mathrm{fm}$ ). The variation of the $R_{s}$ values is dominated by the different single-particle radii obtained from these Skyrme interactions that affects the single-particle cross sections as shown in Fig. 1. From Fig. 7 we find that the theoretical error on the reduction factors is about $15 \%$. This is small compared to the difference between $R_{s}$ for ${ }^{24} \mathrm{Si}-1 p$ and ${ }^{24} \mathrm{Si}-1 n$. When these nuclear matter parameters become better established the results for $R_{S}$ could be reevaluated to reduce the $15 \%$ uncertainty.

\section{SUMMARY}

In summary, we have deduced the reduction of measured spectroscopic strength relative to USDB effective interaction shell-model calculations for two exotic nuclei at the extremes of nuclear binding. The heavy-ion-induced oneproton knockout reactions ${ }^{9} \mathrm{Be}\left({ }^{28} \mathrm{~S},{ }^{27} \mathrm{P}\right) \mathrm{X}$ and ${ }^{9} \mathrm{Be}\left({ }^{24} \mathrm{Si},{ }^{23} \mathrm{Al}\right) \mathrm{X}$ and one-neutron knockout reactions ${ }^{9} \mathrm{Be}\left({ }^{28} \mathrm{~S},{ }^{27} \mathrm{~S}\right) \mathrm{X}$ and ${ }^{9} \mathrm{Be}\left({ }^{24} \mathrm{Si},{ }^{23} \mathrm{Si}\right) \mathrm{X}$ were used to probe weakly bound proton states and strongly bound neutron states at their respective Fermi surfaces. Our results confirm earlier analyses that indicate a stronger reduction in single-particle strength for the strongly bound, deficient nucleon type and a reduction of closer to unity for the weakly bound, excess nucleon type. If the reduction is attributed to correlations missing from truncated-model-space effective-interaction theories, the present work adds support to a scenario in which the valence nucleons of the deficient nucleon species are subject to stronger correlations than those of the excess nucleon species. Uniquely, this effect is accessible to study in exotic nuclei in the vicinity of the drip lines, where these very asymmetric Fermi surfaces are the norm.

\section{ACKNOWLEDGMENTS}

This work was supported by the National Science Foundation under grant nos. PHY-0606007 and PHY-0555366 and the United Kingdom Science and Technology Facilities Council (STFC) under grant no. EP/D003628.
[1] V. R. Pandharipande, I. Sick, and P. K. A. de Witt Huberts, Rev. Mod. Phys. 69, 981 (1997).

[2] W. H. Dickhoff and C. Barbieri, Prog. Part. Nucl. Phys. 52, 377 (2004).

[3] D. Rohe, C. S. Armstrong, R. Asaturyan, O. K. Baker, S. Bueltmann, C. Carasco, D. Day, R. Ent, H. C. Fenker, K. Garrow, A. Gasparian, P. Gueye, M. Hauger, A. Honegger, J. Jourdan, C. E. Keppel, G. Kubon, R. Lindgren, A. Lung, D. J. Mack, J. H. Mitchell, H. Mkrtchyan, D. Mocelj, K. Normand, T. Petitjean, O. Rondon, E. Segbefia, I. Sick, S. Stepanyan, L. Tang, F. Tiefenbacher, W. F. Vulcan, G. Warren, S. A. Wood, L. Yuan, M. Zeier, H. Zhu, and B. Zihlmann and the E97-006 Collaboration, Phys. Rev. Lett. 93, 182501 (2004).

[4] G. J. Kramer, H. P. Blok, and L. Lapikas, Nucl. Phys. A679, 267 (2001).

[5] B. A. Brown, P. G. Hansen, B. M. Sherrill, and J. A. Tostevin, Phys. Rev. C 65, 061601(R) (2002).

[6] J. A. Tostevin, J. Phys. G: Nucl. Part. Phys. 25, 735 (1999).

[7] P. G. Hansen and J. A. Tostevin, Annu. Rev. Nucl. Part. Sci. 53, 221 (2003).

[8] V. Guimarães, J. J. Kolata, D. Bazin, B. Blank, B. A. Brown, T. Glasmacher, P. G. Hansen, R. W. Ibbotson, D. Karnes, V. Maddalena, A. Navin, B. Pritychenko, B. M. Sherrill, D. P. Balamuth, and J. E. Bush, Phys. Rev. C 61, 064609 (2000).

[9] T. Aumann, A. Navin, D. P. Balamuth, D. Bazin, B. Blank, B. A. Brown, J. E. Bush, J. A. Caggiano, B. Davids, T. Glasmacher, V. Guimarães, P. G. Hansen, R. W. Ibbotson, D. Karnes, J. J. Kolata, V. Maddalena, B. Pritychenko, H. Scheit, B. M. Sherrill, and J. A. Tostevin, Phys. Rev. Lett. 84, 35 (2000).

[10] P. G. Hansen and B. M. Sherrill, Nucl. Phys. A693, 133 (2001).

[11] A. Navin, D. Bazin, B. A. Brown, B. Davids, G. Gervais, T. Glasmacher, K. Govaert, P. G. Hansen, M. Hellström, R. W. Ibbotson, V. Maddalena, B. Pritychenko, H. Scheit, B. M. Sherrill, M. Steiner, J. A. Tostevin, and J. Yurkon, Phys. Rev. Lett. 81, 5089 (1998).
[12] V. Maddalena, T. Aumann, D. Bazin, B. A. Brown, J. A. Caggiano, B. Davids, T. Glasmacher, P. G. Hansen, R. W. Ibbotson, A. Navin, B. V. Pritychenko, H. Scheit, B. M. Sherrill, M. Steiner, J. A. Tostevin, and J. Yurkon, Phys. Rev. C 63, 024613 (2001).

[13] J. Enders, A. Bauer, D. Bazin, A. Bonaccorso, B. A. Brown, T. Glasmacher, P. G. Hansen, V. Maddalena, K. L. Miller, A. Navin, B. M. Sherrill, and J. A. Tostevin, Phys. Rev. C 65, 034318 (2002).

[14] A. Gade, D. Bazin, B. A. Brown, C. M. Campbell, J. A. Church, D. C. Dinca, J. Enders, T. Glasmacher, P. G. Hansen, Z. Hu, K. W. Kemper, W. F. Mueller, H. Olliver, B. C. Perry, L. A. Riley, B. T. Roeder, B. M. Sherrill, J. R. Terry, J. A. Tostevin, and K. L. Yurkewicz, Phys. Rev. C 69, 034311 (2004).

[15] A. Gade, D. Bazin, B. A. Brown, C. M. Campbell, J. A. Church, D. C. Dinca, J. Enders, T. Glasmacher, P. G. Hansen, Z. Hu, K. W. Kemper, W. F. Mueller, H. Olliver, B. C. Perry, L. A. Riley, B. T. Roeder, B. M. Sherrill, J. R. Terry, J. A. Tostevin, and K. L. Yurkewicz, Phys. Rev. Lett. 93, 042501 (2004).

[16] J. R. Terry, D. Bazin, B. A. Brown, J. Enders, T. Glasmacher, P. G. Hansen, B. M. Sherrill, and J. A. Tostevin, Phys. Rev. C 69, 054306 (2004).

[17] A. Navin, D. W. Anthony, T. Aumann, T. Baumann, D. Bazin, Y. Blumenfeld, B. A. Brown, T. Glasmacher, P. G. Hansen, R. W. Ibbotson, P. A. Lofy, V. Maddalena, K. Miller, T. Nakamura, B. V. Pritychenko, B. M. Sherrill, E. Spears, M. Steiner, J. A. Tostevin, J. Yurkon, and A. Wagner, Phys. Rev. Lett. 85, 266 (2000).

[18] J. R. Terry, D. Bazin, B. A. Brown, C. M. Campbell, J. A. Church, J. M. Cook, A. D. Davies, D. C. Dinca, J. Enders, A. Gade, T. Glasmacher, P. G. Hansen, J. L. Lecouey, T. Otsuka, B. Pritychenko, B. M. Sherrill, J. A. Tostevin, Y. Utsuno, K. Yoneda, and H. Zwahlen, Phys. Lett. B640, 86 (2006).

[19] A. Gade, D. Bazin, C. A. Bertulani, B. A. Brown, C. M. Campbell, J. A. Church, D. C. Dinca, J. Enders, T. Glasmacher, 
P. G. Hansen, Z. Hu, K. W. Kemper, W. F. Mueller, H. Olliver, B. C. Perry, L. A. Riley, B. T. Roeder, B. M. Sherrill, J. R. Terry, J. A. Tostevin, and K. L. Yurkewicz, Phys. Rev. C 71, 051301(R) (2005).

[20] J. Fridmann, I. Wiedenhöver, A. Gade, L. T. Baby, D. Bazin, B. A. Brown, C. M. Campbell, J. M. Cook, P. D. Cottle, E. Diffenderfer, D. C. Dinca, T. Glasmacher, P. G. Hansen, K. W. Kemper, J. L. Lecouey, W. F. Mueller, H. Olliver, E. Rodriguez-Vieitez, J. R. Terry, J. A. Tostevin, and K. Yoneda, Nature 435, 922 (2005).

[21] J. Fridmann, I. Wiedenhöver, A. Gade, L. T. Baby, D. Bazin, B. A. Brown, C. M. Campbell, J. M. Cook, P. D. Cottle, E. Diffenderfer, D. C. Dinca, T. Glasmacher, P. G. Hansen, K. W. Kemper, J. L. Lecouey, W. F. Mueller, E. Rodriguez-Vieitez, J. R. Terry, J. A. Tostevin, K. Yoneda, and H. Zwahlen, Phys. Rev. C 74, 034313 (2006).

[22] A. Gade, R. V. F. Janssens, D. Bazin, B. A. Brown, C. M. Campbell, M. P. Carpenter, J. M. Cook, A. N. Deacon, D. C. Dinca, S. J. Freeman, T. Glasmacher, M. Horoi, B. P. Kay, P. F. Mantica, W. F. Mueller, J. R. Terry, J. A. Tostevin, and S. Zhu, Phys. Rev. C 74, 047302 (2006).

[23] G. J. Kramer, H. P. Blok, J. F. A. van Hienen, S. Brandenburg, M. N. Harakeh, Y. Y. van der Werf, P. W. M. Glaudemans, and A. A. Wolters, Nucl. Phys. A477, 55 (1988) and references therein.

[24] J. Lee, J. A. Tostevin, B. A. Brown, F. Delaunay, W. G. Lynch, M. J. Saelim, and M. B. Tsang, Phys. Rev. C 73, 044608 (2006).

[25] J. Lee, M. B. Tsang, and W. G. Lynch, Phys. Rev. C 75, 064320 (2007).

[26] J. Enders, T. Baumann, B. A. Brown, N. H. Frank, P. G. Hansen, P. R. Heckman, B. M. Sherrill, A. Stolz, M. Thoennessen, J. A. Tostevin, E. J. Tryggestad, S. Typel, and M. S. Wallace, Phys. Rev. C 67, 064301 (2003).

[27] R. J. Charity, L. G. Sobotka, and W. H. Dickhoff, Phys. Rev. Lett. 97, 162503 (2006).

[28] D. J. Morrissey et al., Nucl. Instrum. Methods B 204, 90 (2003).

[29] D. Bazin et al., Nucl. Instrum. Methods B 204, 629 (2003).

[30] W. F. Mueller et al., Nucl. Instrum. Methods A 466, 492 (2001).

[31] A. Gade, P. Adrich, D. Bazin, M. D. Bowen, B. A. Brown, C. M. Campbell, J. M. Cook, T. Glasmacher, K. Hosier, S. McDaniel, D. McGlinchery, A. Obertelli, L. A. Riley, K. Siwek, and D. Weisshaar, Phys. Rev. C 76, 024317 (2007).

[32] M. Berz, K. Joh, J. A. Nolen, B. M. Sherrill, and A. F. Zeller, Phys. Rev. C 47, 537 (1993).

[33] J. A. Tostevin, Nucl. Phys. A682, 320c (2001).
[34] R. J. Glauber, Lectures in Theoretical Physics (Interscience, New York, 1959), Vol. 1, p. 315.

[35] B. A. Brown, Phys. Rev. C 58, 220 (1998).

[36] B. A. Brown, A. Richter, and R. Lindsay, Phys. Lett. B483, 49 (2000).

[37] B. A. Brown, S. Typel, and W. A. Richter, Phys. Rev. C 65, 014612 (2001).

[38] W. A. Richter and B. A. Brown, Phys. Rev. C 67, 034317 (2003).

[39] C. A. Bertulani and P. G. Hansen, Phys. Rev. C 70, 034609 (2004).

[40] C. A. Bertulani and A. Gade, Comput. Phys. Commun. 175, 372 (2006).

[41] B. A. Brown and W. A. Richter, Phys. Rev. C 74, 034315 (2006).

[42] B. A. Brown, A. Etchegoyen, N. S. Godwin, W. D. M. Rae, W. A. Richter, W. E. Ormand, E. K. Warburton, J. S. Winfield, L. Zhao, and C. H. Zimmerman, Michigan State University, report no. MSU-NSCL 1289 (2004).

[43] G. Audi, A. H. Wapstra, and C. Thibault, Nucl. Phys. A729, 337 (2003).

[44] J. A. Caggiano, D. Bazin, W. Benenson, B. Davids, R. Ibbotson, H. Scheit, B. M. Sherrill, M. Steiner, J. Yurkon, A. F. Zeller, B. Blank, M. Chartier, J. Greene, J. A. Nolen, A. H. Wuosmaa, M. Bhattacharya, A. Garcia, and M. Wiescher, Phys. Rev. C 64, 025802 (2001).

[45] N. Michel, W. Nazarewicz, and M. Ploszajczak, Phys. Rev. C 75, 031301(R) (2007).

[46] N. Michel, W. Nazarewicz, M. Ploszajczak, and K. Bennaceur, Phys. Rev. Lett. 89, 042502 (2002).

[47] C. Mahaux and H. A. Weidenmüller, Shell-Model Approach to Nuclear Reactions (North-Holland, Amsterdam, 1969).

[48] K. Bennaceur, F. Nowacki, J. Okolowicz, and M. Ploszajczak, Nucl. Phys. A651, 289 (1999).

[49] R. Id Betan, R. J. Liotta, N. Sandulescu, and T. Vertse, Phys. Rev. Lett. 89, 042501 (2002).

[50] A. Volya and V. Zelevinsky, Phys. Rev. C 74, 064314 (2006).

[51] D. M. Brink, Phys. Lett. B40, 37 (1972).

[52] B. A. Brown, Phys. Rev. Lett. 85, 5296 (2000); S. Typel and B. A. Brown, Phys. Rev. C 64, 027302 (2001).

[53] J. Bartel, P. Quentin, M. Brack, C. Guet, and M. B. Hakannsson, Nucl. Phys. A396, 79 (1982).

[54] B. A. Brown, G. Shen, G. C. Hillhouse, J. Meng, and A. Trzcinska, Phys. Rev. C 76, 034305 (2007).

[55] E. Chabanat, P. Bonche, P. Haensel, J. Meyer, and T. Schaeffer, Nucl. Phys. A635, 231 (1998).

[56] S. Goriely, M. Samyn, J. M. Pearson, and M. Onsi, Nucl. Phys. A750, 425 (2005). 